OPEN

SUBJECT AREAS:

BREEDING

AGRICULTURAL GENETICS

Received

6 January 2014

Accepted

14 February 2014

Published

3 March 2014

Correspondence and requests for materials should be addressed to

Z.Q.L. (zqliu@scau. edu.cn) or G.Q.Z. lgqzhang@scau.edu.

cn)

\title{
Characterization of Epistatic Interaction of QTLs LH8 and EH3 Controlling Heading Date in Rice
}

Jingbin Chen ${ }^{1,2}$, Xiaoyan Li1,3, Cheng Cheng', Yahuan Wang', Mao Qin', Haitao Zhu', Ruizhen Zeng', Xuelin Fu', Ziqiang Liu' \& Guiquan Zhang'

\footnotetext{
'State Key Laboratory for Conservation and Utilization of Subtropical Agro-Bioresources, Guangdong Key Laboratory of Plant Molecular Breeding, College of Agriculture, South China Agricultural University, Guangzhou 510642, China, ${ }^{2}$ Institute of Vegetable Crops, Jiangsu Academy of Agricultural Sciences, Nanjing 210014, China, ${ }^{3}$ College of Agronomy, Northwest Agriculture \& Forestry University, Yangling, 712100, China.
}

Heading date is a critical trait for adaptation of rice to different cultivation areas and cropping seasons. We evaluated the heading dates of 1,123 chromosome segments substitution lines (CSSLs) in the genetic background of an elite rice variety Huajingxian74 (HJX74). A CSSL with the substituted segments from Zihuil00 exhibited late heading under both natural long-day (NLD) and natural short-day (NSD) conditions, and the late heading phenotype was controlled by two novel epistatic loci on chromosome 8 and chromosome 3, respectively, termed $L H 8$ and $E H 3$. The function of $E H 3$ was dependent on the $L H 8$ genotype through epistatic interaction between $E H^{Z^{\text {zihuiloo }}}$ and $L H 8^{\text {zihuiloo }}$ alleles. Genetic and molecular characterization revealed $\mathrm{LH} 8$ encodes a CCAAT-box-binding transcription factor with Heading date 1 (Hd1)-binding activity and may delay flowering by repressing the expression of Early heading date1 (Ehd1). Our work provides a solid foundation for further study on gene interaction in heading date and has application in breeding rice with greater adaptability.

ice is a short-day (SD) plant, and is becoming an increasingly important model monocot plant for molecular biological study on agronomic important traits such as heading date. Heading date, flowering time in cereal crops, is a critical agronomical determinant for adaptation to specific cropping locations and growing seasons for current varieties of cultivated rice and is an important consideration for rice breeders. The molecular and developmental determinants of heading date have thus particularly important genetic targets for domestication and for breeding new varieties of rice.

The timing of flower initiation is controlled by both environmental and internal signals ${ }^{1,2}$. By taking advantage of pioneering works in long-day (LD) plant Arabidopsis, many molecular components of rice flowering process initiated from photoperiod and light signals have been identified through natural variations, quantitative trait locus (QTL) analyses and by screening mutant populations (as summarized and reviewed $\mathrm{in}^{3}$ ). Two independent flowering pathways, the conserved $H d 1$-dependent, and unique Ehd1-dependent pathways, control heading time in rice ${ }^{4-7}$. The two pathways function redundantly under SD conditions but antagonistically under LD conditions. ${ }^{8}$. Hd1, a rice ortholog of Arabidopsis CONSTANS (CO) that is regulated by OsGI (a rice ortholog of Arabidopsis GIGANTEA), activates flowering under SD conditions and represses it strongly under LD conditions through regulating the expression of Heading date3a ( $\mathrm{Hd} 3 \mathrm{a}$ ), a rice ortholog of Arabidopsis florigen FLOWERING LOCUS T $(F T)^{4,9}$. Ehd 1 encodes a B-type response regulator that is highly conserved in cultivated rice, but has no homolog in Arabidopsis ${ }^{6}$. Ehd 1 can promote flowering by activating the expression of $H d 3 a$ and RICE FLOWERING LOCUS T1 (RFT1), the closest paralog of $H d 3 a^{6,7,10}$. The expression of Ehd 1 is promoted by a number of positive regulators, including RID1 (also known as OsID1 and Ehd2) ${ }^{11-13}$, Early heading date $3(\text { Ehd } 3)^{14}$, Early heading date4 (Ehd4) ${ }^{15}$, OsMADS5 $1^{16}$ and OsMADS50 (also known as DTH3) ${ }^{17-19}$. On the other hand, several factors repress the expression of $E h d 1$, thereby delaying flowering, such as $G h d 7^{20}, D T H 8$ (also known as Ghd8 and LHD1 $)^{21-23}, S E 5^{24}, O s C O L 4^{25}$ and OsLFL $1^{26,27}$. Therefore, Ehd 1 serves as a critical convergence point of regulation by multiple signaling pathways. The flowering signals are collected from $H d 1$ and Ehd1, and are transducted to a pair of florigen genes, $H d 3 a$ and RFT1, promoting heading under SD and LD conditions, respectively ${ }^{7,10}$. After production in leaves, these florigens move to the shoot apical meristem and promote floral transition by activating the expression of OsMADS14 and OsMADS15. Although these studies have revealed 
much insight into the flowering control of rice, the underlying molecular mechanisms, especially the genetic interactions among these factors, are still not well understood.

The advantages of CSSLs in precise and detailed phenotype evaluation, minor QTL detection and validation of gene-gene and geneenvironment interactions facilitated both genetic studies and rice improvement ${ }^{28-30}$. Therefore, we had constructed a library of 1,123 CSSLs in rice using HJX74, an elite indica variety from south China, as recipient and 24 accessions, including 14 indica and 10 japonica, collected worldwide as donors to understand the molecular mechanism of agronomic traits including heading date ${ }^{31,32}$. Each CSSL contains a very small number of well-characterized chromosome segments from one of the 24 donor varieties.

In this study, we found a CSSL with the substituted segments from Zihui100 exhibited late heading under both NLD and NSD conditions, and the late heading phenotype was controlled by two novel epistatic loci on chromosome 8 and chromosome 3, respectively, termed $L H 8$ and $E H 3$. Map-based cloning of $L H 8$ revealed it encodes a CCAAT-box-binding transcription factor and is allelic to DTH8. Molecular function of $\mathrm{LH} 8$ was further studied by expression analysis and yeast-two-hybrid experiment.

\section{Results}

Identification of a CSSL with late heading. The procedure for the development of the CSSLs was summarized in Fig. 1. HJX74 (as the female parent) was crossed with one of the 24 donors (Zihui100 was taken as an example), and the $\mathrm{F}_{1}$ plants were backcrossed with HJX74 to develop the $\mathrm{BC}_{1} \mathrm{~F}_{1}$ generation. Polymorphic SSR markers were used in the selection of the donor chromosomal segments. Using the same method, $\mathrm{BC}_{6} \mathrm{~F}_{1}$ plants were obtained, and were selfcrossed to produce $\mathrm{BC}_{6} \mathrm{~F}_{2}$ lines in which the majority of genomic regions were homozygous for HJX74 alleles. Totally, 1,123 such CSSLs in the HJX74 genetic background were developed ${ }^{31,32}$. To further understand how flowering in rice was controlled, we evaluated the heading date of the 1,123 CSSLs under natural growth conditions. A CSSL, CSSL5, with substituted segments from Zihui100 exhibited late heading compared with the recipient HJX74 under both NLD $(114.4 \pm 1.4 \mathrm{~d}$ for CSSL5, $100.1 \pm 0.5 \mathrm{~d}$ for
HJX74) and NSD conditions (84.5 $\pm 1.6 \mathrm{~d}$ for CSSL5, $76.7 \pm 1.4 \mathrm{~d}$ for HJX74) in 2009 (Fig. 2a and 2b).

To analyze the genetic basis for the late heading of CSSL5, we crossed CSSL5 with the recipient HJX74 (Fig. 1). 73 out of $326 \mathrm{~F}_{2}$ plants showed heading date later than that of CSSL5 $(113.0 \pm 1.3 \mathrm{~d})$ under NLD conditions in 2010, and the whole population exhibited trimodal distribution of heading date fitting $4: 9: 3$ segration ratio $\left(\chi^{2}\right.$ $\left.=4.67<\chi_{0.05,2}^{2}=5.99\right)$ (Fig. $2 \mathrm{c}$ ). These results indicate that the late heading phenotype was controlled by two genes with negative epistasis.

Confirmation of the two genes by resequencing. To delimit the heading date genes on the substituted segments, CSSL5 and HJX74 were high-throughput genotyped by whole-genome resequencing, and an ultrahigh-quality physical map was constructed (Fig. 3). 11 substituted segments with a total length of $20.64 \mathrm{Mb}$ derived from Zihui100 were found to be distributed over 6 chromosomes (Fig. 3 and Supplementary Table S1 online). Molecular markers were subsequently designed on the substituted segments, and were used to analyze their linkage with heading date phenotype of the $F_{2}$ population. Two QTLs were identified on chromosome 3 and 8, respectively, designated as $E H 3$ and $L H 8$, respectively. $E H 3$ was mapped in the $750 \mathrm{~kb}$ genomic region between the Id32 and Id33 markers and had the contributions to phenotypic variation by $14.0 \%$, the allele from Zihuil 100 could shorten the heading date by $2.32 \mathrm{~d}$ and the dominant effect was -3.24. LH8 was mapped in the $460 \mathrm{~kb}$ genomic region between the Id83 and Id82 markers and had the contributions to phenotypic variation by $65.3 \%$. The allele from Zihui100 could delay the heading date by $6.62 \mathrm{~d}$ and the dominant effect was 3.44 (Table 1).

Epistatic interaction between $\mathbf{E H 3}$ and $\mathbf{L H 8}$. To examine the genetic interactions between $E H 3$ and $L H 8$, we scored heading dates in the $\mathrm{F}_{2}$ population under NLD and NSD conditions in 2011 (Fig. 4). The $\mathrm{F}_{2}$ plants were classified into nine genotype classes based on the EH3 and LH8 alleles, and we compared the average heading date among the nine classes. Under NLD conditions, two-way ANOVA revealed the existence of digenic

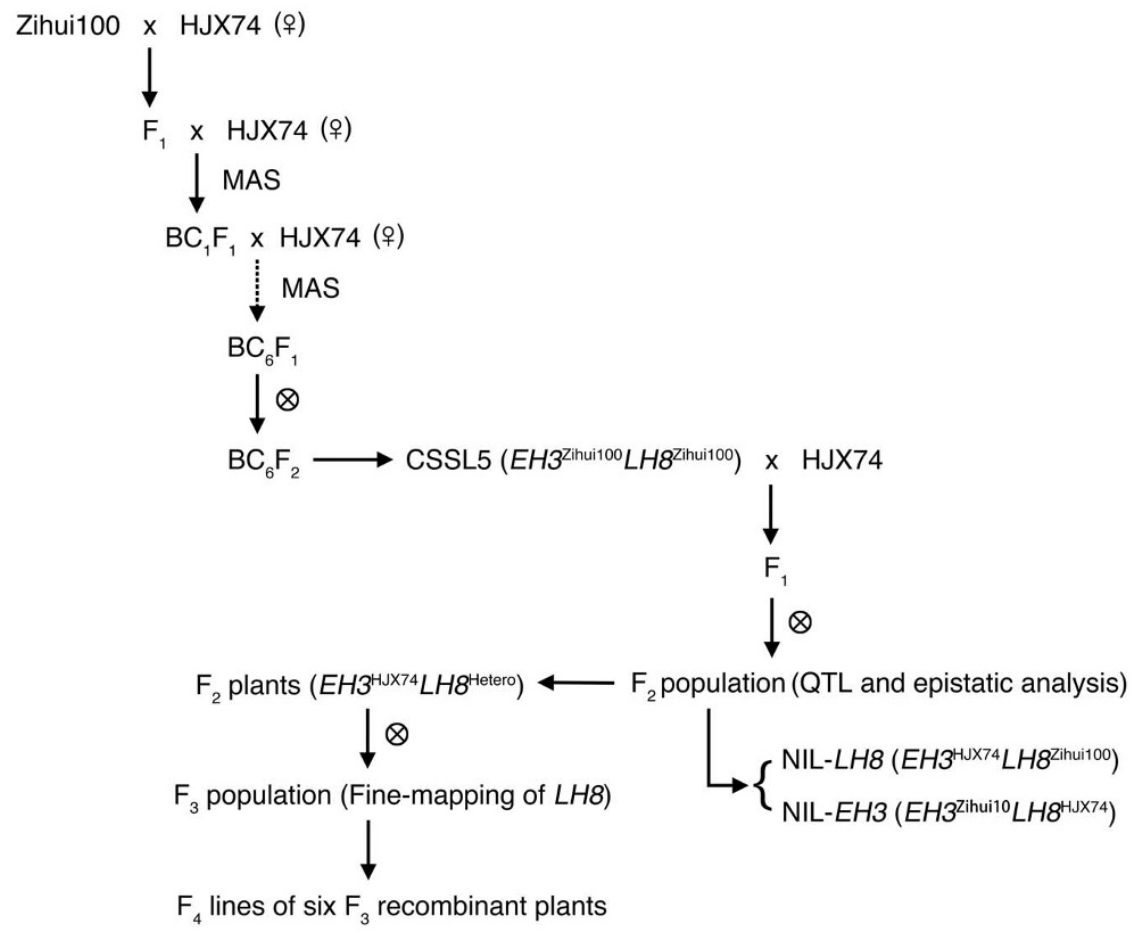

Figure 1 Flowchart of the development of CSSLs and QTL analysis. MAS: marker-assisted selection. 


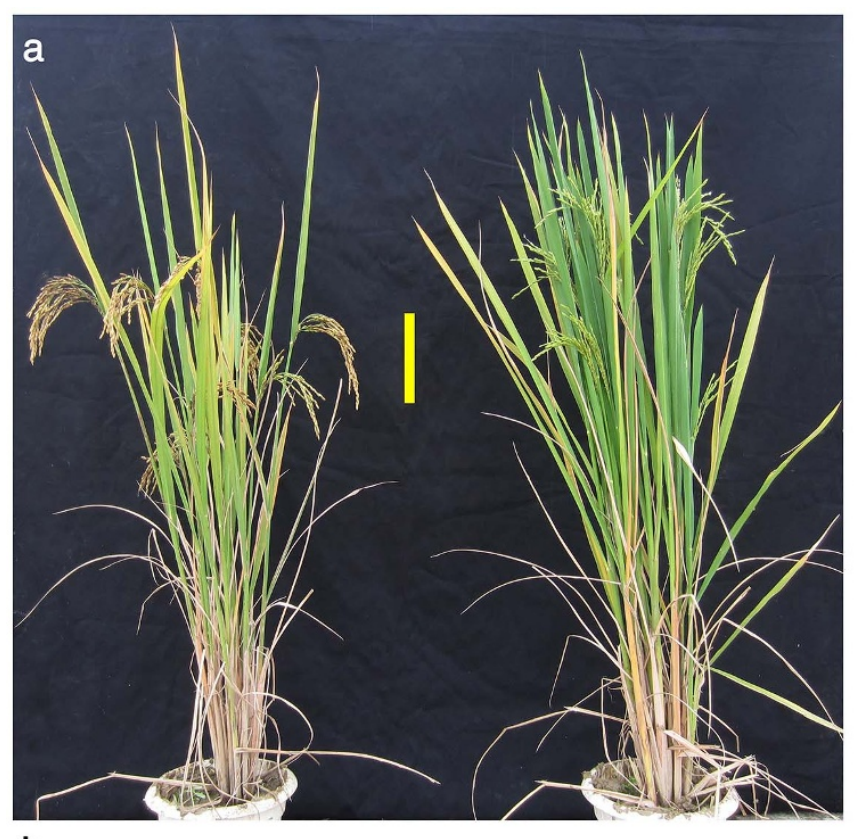

b

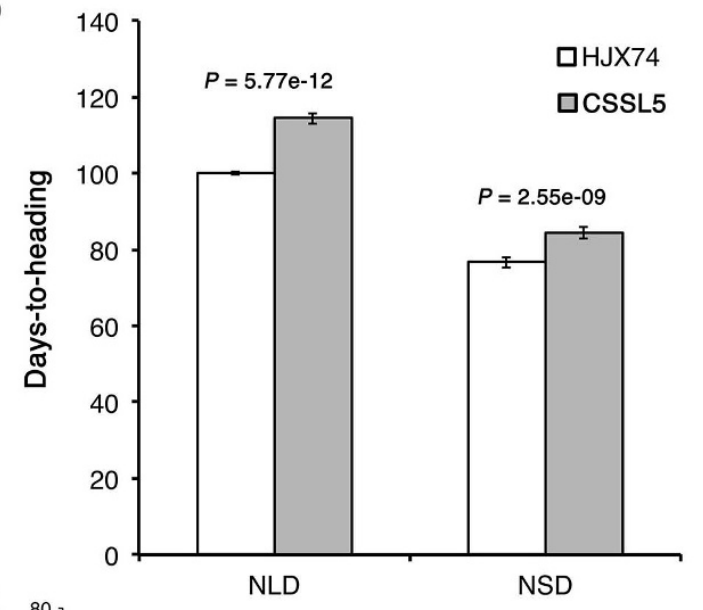

C

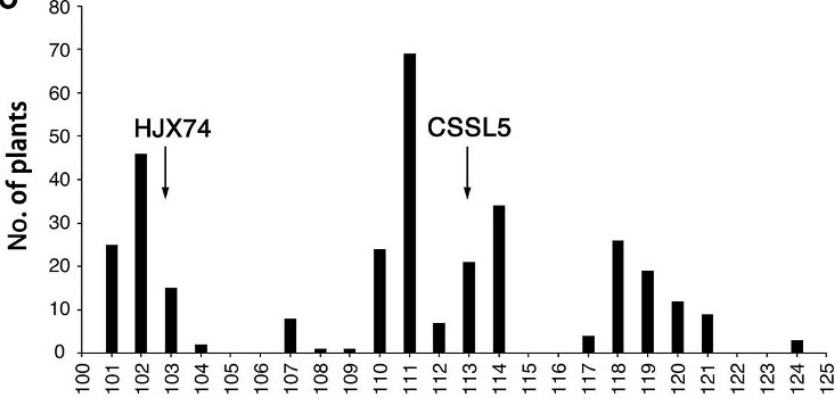

Days-to-heading

Figure $2 \mid$ The heading dates of HJX74, CSSL5 and their $\mathrm{F}_{2}$ population. (a). Phenotypes of HJX74 (left) and CSSL5 (right). Yellow bar $=15 \mathrm{~cm}$. (b). Days-to-heading in HJX74 and CSSL5 in different photoperiodic conditions. NLD, natural long-day conditions; NSD, natural short-day conditions. Each column represents mean \pm s.d. $(n=30)$. (c). Frenquency distributions of days-to-heading in an $\mathrm{F}_{2}$ population from HJX74 x CSSL5 under NLD conditions. Black arrows indicate the mean DTH of HJX74 and $\operatorname{CSSL5}(\mathrm{n}=30)$, respectively.

interaction between $E H 3$ and $L H 8(P=5.96 \mathrm{e}-06)$. Orthogonal contrast test showed that AA (Additive by additive), but not $\mathrm{AD}$ (Additive by dominance), DA (Dominance by additive) or DD (Dominance by dominance) type of interaction was significant in the interaction of $E H 3$ and $L H 8(P<0.0001)$. The effect of the
Zihui100 allele at LH8 (i.e., increased days-to-heading (DTH)) was regardless of the allele status of $E H 3$. In comparison, the effect of the Zihui100 allele at EH3 (i.e., shortened DTH) was observed in two genotype classes, homozygous for the Zihuil00 allele at the LH8 locus and heterozygous, but not in the class homozygous for the HJX74 allele at the LH8 locus (Fig. 4a). Under NSD conditions, the Zihuil00 allele at $\mathrm{LH} 8$ was also observed to delay heading regardless of the allele status of $E H 3$. However, the two-way ANOVA suggested the digenic interaction between $\mathrm{EH} 3$ and $\mathrm{LH} 8$ was not significant under NSD conditions $(P=0.883)$ (Fig. $4 \mathrm{~b})$.

Map-based cloning of $\mathbf{L H 8}$. To fine map the LH8 gene, we selected $\mathrm{F}_{2}$ plants with homozygous $E H 3$ allele from $\mathrm{HJX} 74$ and heterozygous LH8 allele from Zihui100 to generate $F_{3}$ population (Fig. 1). $F_{3}$ population showed bimodal distribution for DTH fitting 3:1 segregation ratio under both NLD and NSD conditions in $2012\left(\chi^{2}\right.$ $=0.04<\chi_{0.05,1}^{2}=3.84$ and $\chi^{2}=0.03<\chi_{0.05,1}^{2}=3.84$ for NLD and NSD, respectively), indicating the Zihui100 allele at LH8 increased DTH in a dominant manner (Fig. 5a and 5b). 7 new markers were developed in the marker interval of Id83-Id82, and were used to analyze a total of 2,159 $\mathrm{F}_{3}$ plants. 6 recombinants were identified between markers RM22475 and RM25 (Fig. 5c). The selfpollinated progeny $\left(\mathrm{F}_{4}\right.$ lines) of those 6 plants were used to determine the genotypes of LH8 (Fig. 1). The recombinants $\mathrm{r} 2$ and r5 restricted $L H 8$ to the $28.3 \mathrm{~kb}$ genomic region between the Id 87 and Id811 markers, including 3 putative genes, a transferase family protein (LOC_Os08g07730), a SERK-family receptor-like protein kinase (LOC_Os08g07760) and a CCAAT-box-binding transcription factor (LOC_Os08g07740) which had been reported to function in heading date control in rice (Fig. 5c). The sequencing of $\mathrm{LH} 8$ genomic regions from CSSL5 and HJX74 revealed two GGC insertion and a 1,116-bp deletion in LOC_Os08g07740 of HJX74 (Fig. 6a), resulting in a 2-glycine insertion in the middle and a big alternation in the $\mathrm{C}$-terminal region of the translated protein, respectively (Fig. 6b). These results suggest that LOC_Os08g07740 corresponds to LH8 controlling the late heading in CSSL5.

Characterization of $\mathbf{L H 8}$ in rice. To examine whether the sequence mutations can alter the expression of $\mathrm{LH} 8$, we examined the expression levels of LH8 in CSSL5, HJX74 and NIL-LH8 (a line selected from $\mathrm{LH} 8 \mathrm{~F}_{2}$ segragating population with homozygous EH3 allele from $\mathrm{HJX74}$ and homozygous LH8 allele from Zihui100) under NSD conditions in 2013. No significant difference in the LH8 expression levels could be detected among the three materials, suggesting that late heading did not result from the LH8 transcription. The expression levels of other heading date genes were also analyzed. For $H d 1$ and OsMADS50, no difference could be detected among the three materials under NSD conditions. The expression levels of Ehd1, Hd3a and RTF1 were less in NIL-LH8 than those in HJX74 under NSD conditions, indicating that LH8 may delay heading through suppressing the expressions of these floral transition activators. In contrast, the expression levels of Ehd1 and RTF1 were higher in CSSL5 than those in HJX74. The different expression levels of Ehd1 and RFT1 between CSSL5 and NIL-LH8 may result from the function of different $E H 3$ alleles (Fig. 7a).

To study the involvement of the 2-glycine indel and the C-terminal region of the LH8 protein in its function, we tested the interaction between LH8 and Hd1 by yeast-two-hybrid method. Yeast cells that coexpressed the $\mathrm{LH} 8^{\text {Zihuiloo }}$ bait and Hd1 prey fusion proteins were able to grow on media lacking histidine and adenine, indicating that these proteins can bind each other in yeast cells to promote the expression of the HIS3 and ADE2 reporter genes. However, the similar interaction could not be detected between $\mathrm{LH} 8^{\mathrm{HJX74}}$ and $\mathrm{Hd} 1$, indicating the 2-glycine indel and the C-terminal 


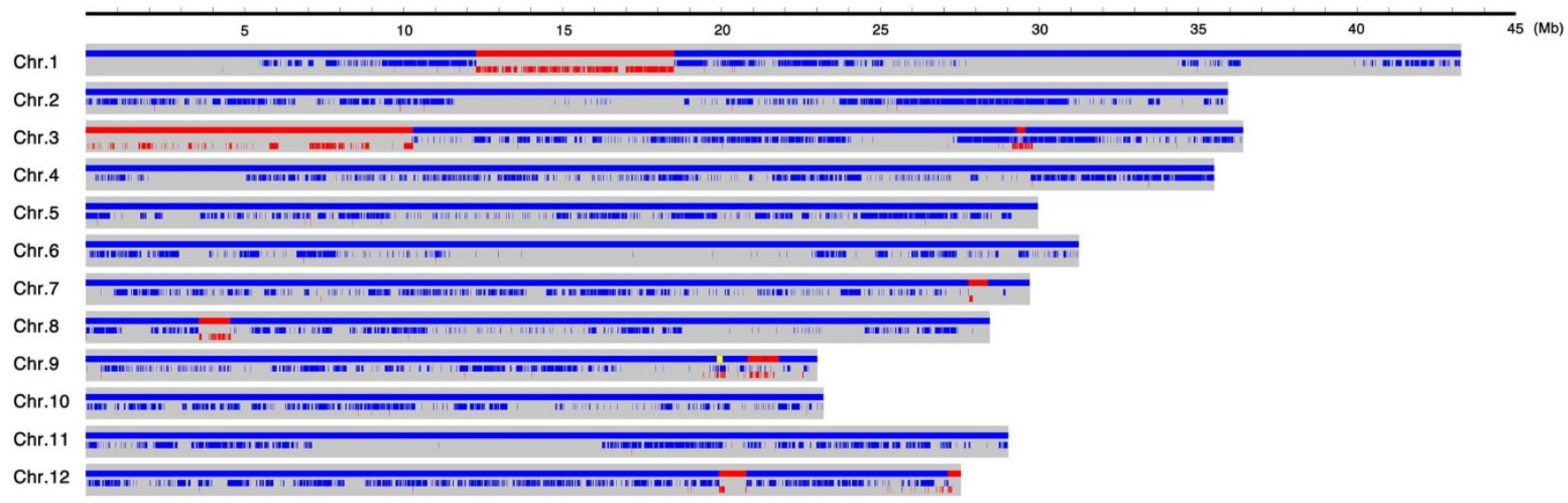

Figure $3 \mid$ Physical map of CSSL5 based on the whole-genome resequencing data. Red areas indicated the substituted chromosome segments from Zihui100, while the blue areas indicated chromosome regions of HJX74. The tiny yellow area on chromosome 9 indicated a heterozygous segment between HJX74 and Zihuil00.

Table 1 Heading date QTLs detected in an $\mathrm{F}_{2}$ population from HJX74 x CSSL5 under NLD conditions

\begin{tabular}{lccrcrr} 
QTL & Chromosome & $\begin{array}{c}\text { Marker } \\
\text { interval }\end{array}$ & LOD $^{\mathrm{a}}$ & PVE $^{\mathrm{b}}$ & \multicolumn{1}{c}{$\mathrm{a}^{\mathrm{c}}$} & \multicolumn{1}{c}{$\mathrm{d}^{\mathrm{d}}$} \\
\hline EH3 & 3 & Id32-ld33 & 4.13 & $14.0 \%$ & -2.32 & -3.24 \\
LH8 & 8 & Id83-ld82 & 53.12 & $65.3 \%$ & 6.62 & 3.44 \\
\hline
\end{tabular}

aLog-likelihood value.

bPercentage phenotypic variance explained by the QTL.

'Additive effect of the Zihui 100 allele on days to heading

dDominant effect of the Zihui 100 allele.

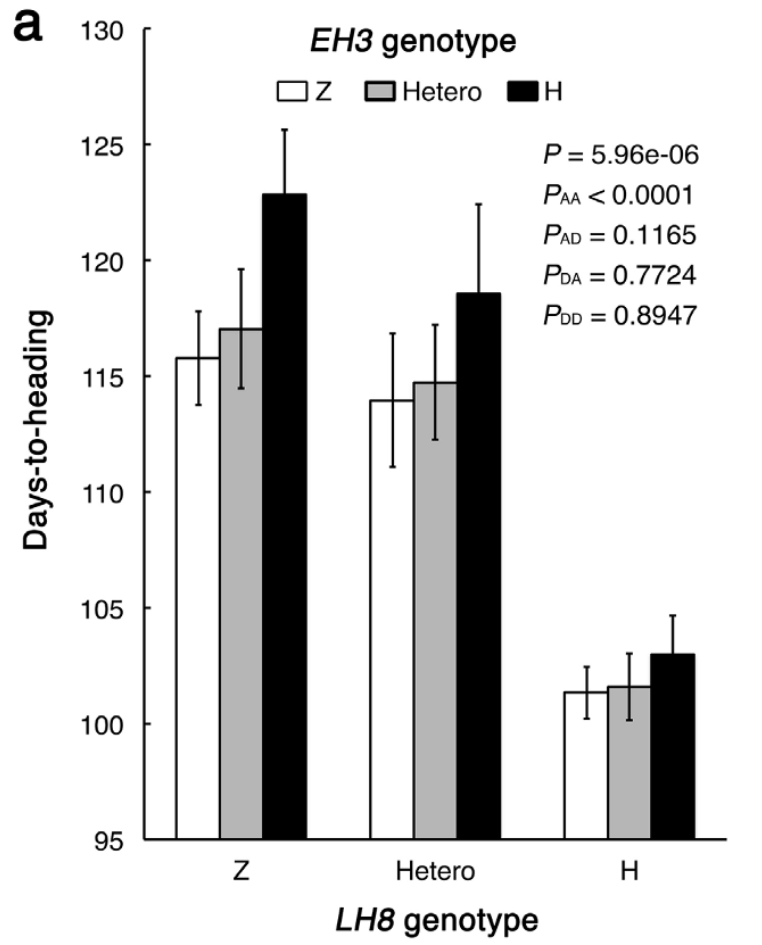

region of LH8 might be important for its interaction with Hd1 (Fig. 7b).

\section{Discussion}

Until now, a total of 734 rice heading date QTLs have been reported (http://www.gramene.org/qtl), and some of them have been molecularly characterized ${ }^{3}$. Besides understanding the biological function of single gene/QTL, clarification of genetic interactions among these genes/QTLs is also important. Actually, several examples of epistatic interactions among heading date genes/QTLs have been reported $^{33-37}$. By eliminating genetic background noises, CSSLs offer a practical solution to bridge the huge gap of knowledge between the

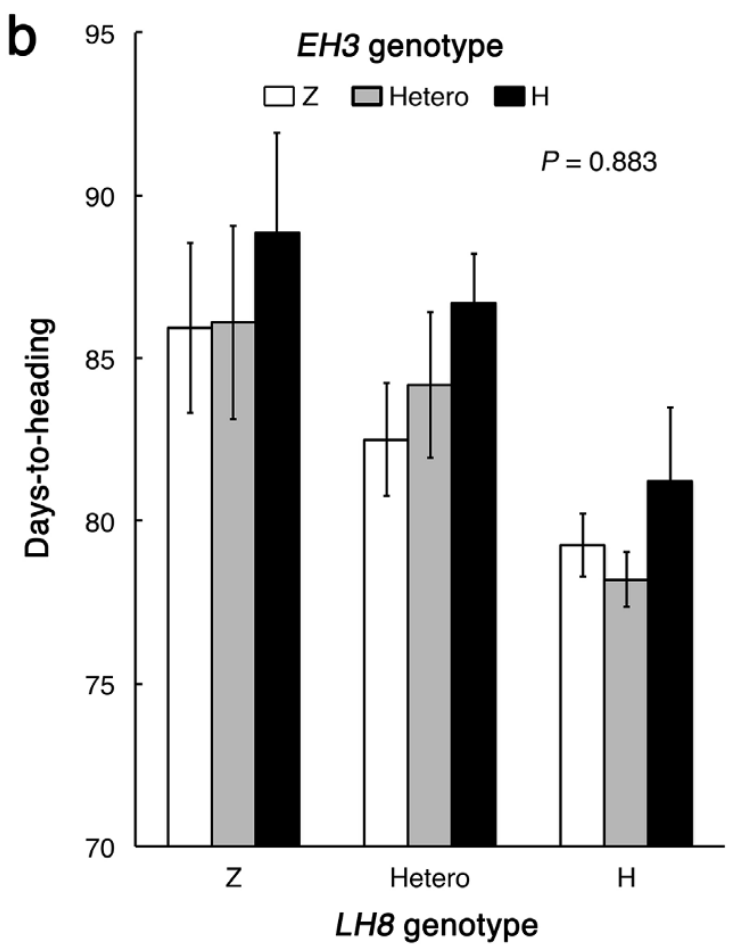

Figure $4 \mid$ Epistatic interaction between $L H 8$ and $E H 3$. Differences in days-to-heading for nine genotype classes for combinations of $L H 8$ and $E H 3$ under NLD (a) and NSD (b) conditions in the $\mathrm{F}_{2}$ populations. Genotypes were determined using the closely linked markers Id83-Id82 (LH8) and Id32Id33 (EH3). Z, Hetero and $\mathrm{H}$ indicate homozygous for Zihui100 allele, heterozygous and homozygous for the HJX74 allele, respectively. $P_{\mathrm{AA}}$, $P_{\mathrm{AD}}, P_{\mathrm{DA}}$ and $P_{\mathrm{DD}}$ are significant probalities for $\mathrm{AA}, \mathrm{AD}, \mathrm{DA}$ and $\mathrm{DD}$ interactions between $L H 8$ and $E H 3$, respectively. 


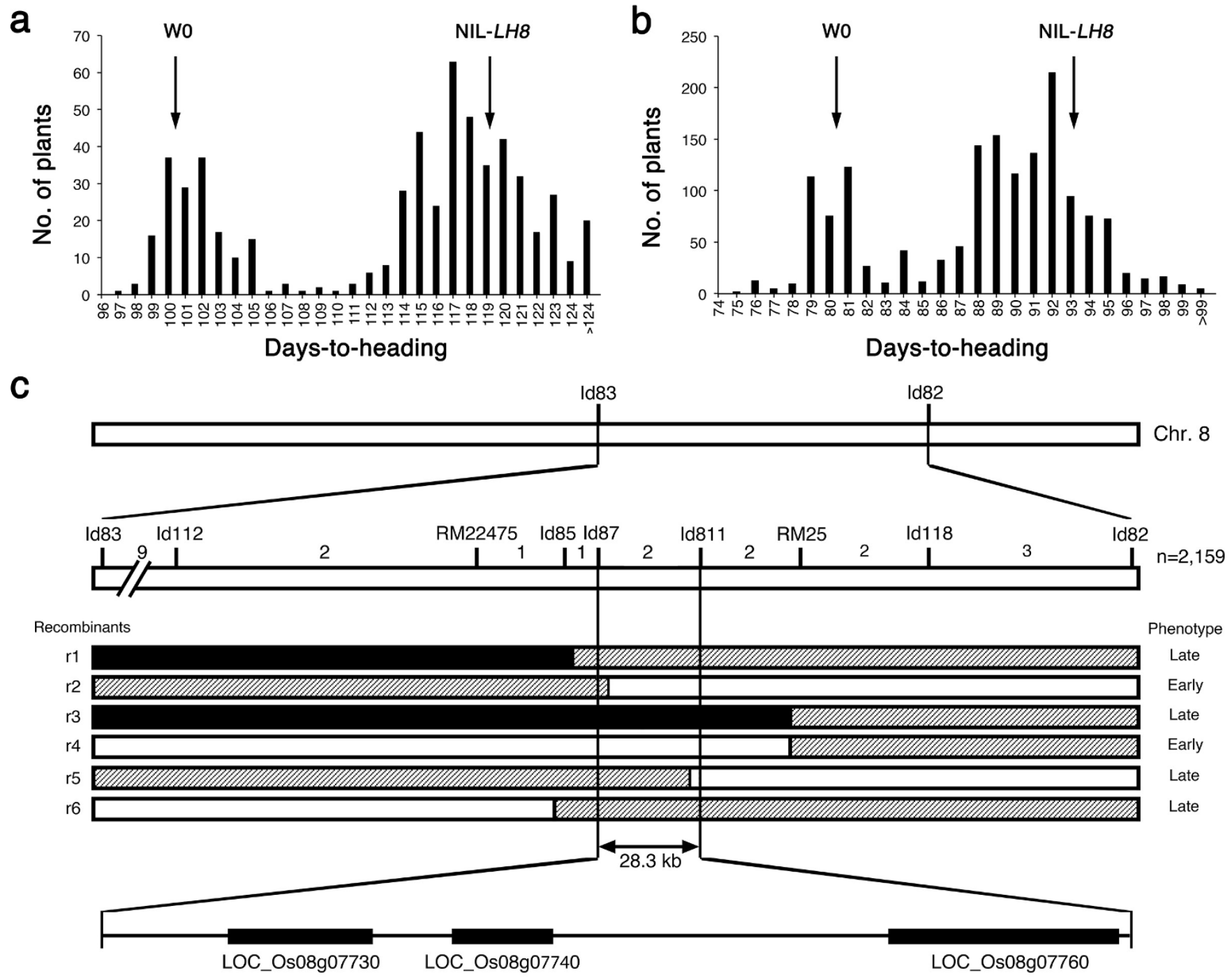

Figure 5 Map-based cloning of the $\mathbf{L H} 8$ gene in rice. (a and b). Frenquency distributions of days-to-heading in an $\mathrm{F}_{3}$ population from HJX74 x CSSL5 under NLD (a) and NSD (b) conditions. Black arrows indicate the mean DTH of HJX74 and NIL-LH8 ( $\mathrm{n}=30$ ), respectively. (c). High-resolution mapping of $L H 8$. The genetic map of $L H 8$ was based on recombinant events among 2,159 $\mathrm{F}_{3}$ plants from HJX74 x CSSL5. The number of recombinants between adjacent markers is shown above the bar. White, shadow and black regions shown in the recombinants indicate homozygous regions for HJX74 allele, heterozygous and homozygous regions for the Zihui100 allele, respectively.

genotype and phenotype, and therefore, are especially useful in studying complicated traits including heading date. In this study, a novel epistatic interaction between $\mathrm{LH} 8$ and $\mathrm{EH} 3$ involved in flowering control in rice was detected. Under NLD conditions and HJX74 genetic background, in plants homozygous for the $L H 8^{\mathrm{HJX} 74}$ allele, difference in DTH between $E H 3^{\text {Zihuilo0 }}$ and $E H 3^{\mathrm{HJX} 74}$ alleles was small (about 1 day), as shown in HJX74 and NIL-EH3 (a line selected from $L H 8 \mathrm{~F}_{2}$ segragating population with homozygous $L H 8$ allele from HJX74 and homozygous EH3 allele from Zihui100) (Fig. 8a). A large effect of $E H 3^{\text {Zihuiloo }}$ allele on heading promotion (about 7 days) occurred with the $L H 8^{\text {Zihuiloo }}$ allele, indicating $E H 3^{\text {Zihuiloo }}$ allele could function to shorten heading date only when $L H 8^{\text {Zihuiloo }}$ allele was concurrently present, and therefore, the function of EH3 was dependent on the LH8 genotype through epistatic interaction between $E H 3^{\text {Zihuil00 }}$ and $L H 8^{\text {Zihuiloo }}$ alleles (Fig. 8a). This result suggests that $E H 3$ might function as a modifier of LH8. Genetic interaction between $\mathrm{LH} 8$ and $E H 3$ suggested that QTL pyramiding would be an effective method for the development of varieties with desirable heading dates under different growth conditions.

To facilitate the identification of the location of $\mathrm{LH} 8$ and $\mathrm{EH} 3$, the high-throughput genotyping technology was exploited. The recipi- ent HJX74 and CSSL5 were resequenced at the sequencing depth of 66- and 1-fold, respectively. Besides a large substituted segment from the short arm end to RM282 on chromosome 3 which was detected through traditional marker-assisted selection during CSSLs construction $^{31}, 10$ additional substituted segments were identified distributing over 6 chromosomes, including some small introgressed segments that might be difficult to be detected by SSR markers (see Supplementary Table S1 online). Indeed, LH8 was found on untargeted chromosomal region on chromosome 8 . These results showed that the high-throughput genotyping method by resequencing has a higher accuracy than marker-assisted selection. Owning to the deep sequencing coverage of the recipient HJX74, resequencing the CSSL5 with genome coverage as low as 1-fold was enough to rapidly validate and delimit the QTL, suggesting the efficiency of this mapping-bysequencing method in discovering the genes responsible for quantitative trait loci. Our results presented a good example of combining the advanced material with uniform genetic background and highthroughput resequencing technology in economically accelerating gene identification.

The sequencing of LH8 genomic regions from CSSL5 and HJX74 revealed two GGC insertion and a 1,116-bp deletion in 


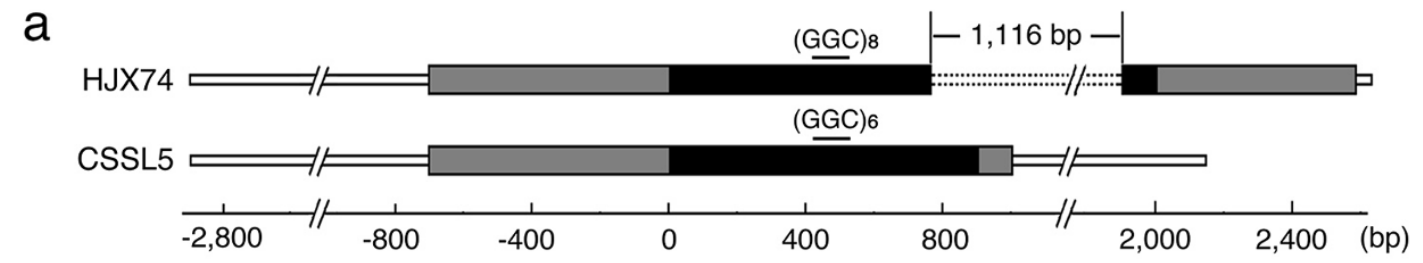

b
HJX74
1 MKSRKSYGHLLSPVGSPPSDNESGAAAAAAAAGGGGCGSSAGYVVYGGGGGGDSPAKEQD
1 MKSRKSYGHLLSPVGSPPSDNESGAAAAAAAAGGGGCGSSAGYVVYGGGGGGDSPAKEQD

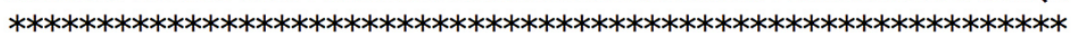

HJX74 61 RFLPIANVSRIMKRSLPANAKISKEAKETVQECVSEFISFVTGEASDKCQREKRKTINGD 120

CSSL5 61 RFLPIANVSRIMKRSLPANAKISKEAKETVQECVSEFISFVTGEASDKCQREKRKTINGD 120

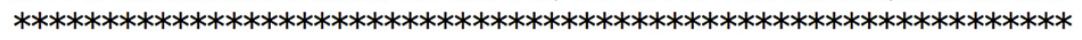

HJX74 121 DLLWAMTTLGFEAYVGPLKSYLNRYREAEGEKAAVLGGAGGAAAARHGEGGCCGGGGGGG 180 CSSL5 121 DLLWAMTTLGFEAYVGPLKSYLNRYREAEGEKAAVLGGAGGAAAARHGEGGCCGGGGG-- 178

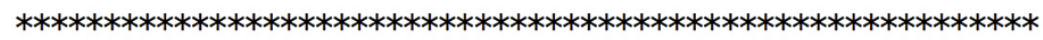

HJX74 181 ADGVVIDGHSPLAGGLSHSHHGHQQQDGGGDVGLMMGGGAAGVGYNAGAGSTTTAFYAPA 240 CSSL5 179 ADGVVIDGHSPLAGGLSHSHHGHQQQDGGGDVGLMMGGGAAGVGYNAGAGSTTTAFYAPA 238 ************************************************************************

\section{HJX74 241 ATAASGNKAYCGGDGSRVMEFEGIIVALYT------AWRMLLYCPFSLPLLWLHA--- 289 CSSL5 239 ATAASGNKAYCGGDGSRVMEFEGIGGEEESGGGGGGGERGFAGHLHGVQWFRLKRSTN 296 $* * * * * * * * * * * * * * * * * * * * * * * * \quad . \quad * \quad *$.}

Figure 6 Sequence comparision of $L H 8$ from HJX74 and CSSL5. (a). Structure of $L H 8$ in HJX74 and CSSL5. Black and gray regions represent the ORF and UTR regions of $L H 8$, respectively. The dotted line represents the $1,116 \mathrm{bp}$ deletion in HJX74. (b). Protein alignment of LH8 from the predicted protein of HJX74 and CSSL5 using the EMBL software ClustalW2 multiple sequence alignment tool.

LOC_Os08g07740 of HJX74, resulting in a 2-glycine insertion in the middle and a big alternation in the C-terminal region of the translated protein, respectively (Fig. 6). Such sequence variations in the coding region of LOC_Os08g07740 between CSSL5 and HJX74 suggested that LOC_Os08g07740 might correspond to LH8 controlling the late heading in CSSL5 and NIL-LH8. LOC_Os08g07740 encodes a CCAAT-box-binding transcription factor which had been reported to strongly inhibit floral transition with names of DTH8, Ghd8 and LHD $1^{21-23}$. Similar to DTH8 and Ghd8, LH8 increased plant height and grain number per panicle under NLD conditions (Fig. 2 and Supplementary Table S2 online), indicating LH8 is allelic to DTH8, Ghd8 and LHD1. Indeed, the two GGC insertion and one 1,116-bp deletion in the coding region of LOC_Os08g07740 of HJX74 had also been found in the ZS97 allele at Ghd $8^{22}$. However, unlike DTH8 and LHD1 delaying flowering only under NLD/LD conditions ${ }^{21,23}$ and Ghd8 functioning oppositely under SD and LD conditions ${ }^{22}$, LH8 delays the heading date under both NSD and NLD conditions. These differences in function may come from different gene-background interactions or different growth conditions. Therefore, genes controlling important agronomic traits are necessary to be further studied in different genetic backgrounds and under different natural field conditions to give a full understanding of its effects and to offer practical instructions in rice breeding.

Expression analysis revealed the transcripts of several flowering promoting factors including Ehd1, $H d 3 a$ and RFT1 were fewer in NIL-LH8 than those in HJX74 under NSD conditions, suggesting LH8 might delay flowering by down-regulating the expression of Ehd1, Hd3a and RFT1 (Fig. 8b). The expressions levels of $H d 1$ and OsMADS50 showed no significant differences among HJX74, CSSL5 and NIL-LH8 under NSD conditions, suggesting they might function upstream of $L H 8$ or parallel to $L H 8$. Furthermore, no significant difference of $\mathrm{LH} 8$ expression were found among HJX74, CSSL5 and NIL-LH8 under NSD conditions, indicating the reason for the change of heading date was not caused by LH8 expression, but by nucleotide changes of the coding sequence. The 6-bp and 1,116-bp indels in the coding region of $L H 8$ resulted in a big sequence alternation of the translated protein, especially in the C-terminal region.

The protein encoded by $\mathrm{LH} 8$ is a putative HAP3 subunit of the HAP complex, which binds to the CCAAT box, a cis-acting element present in approximately $25 \%$ of eukaryotic gene promoters ${ }^{38,39}$. HAP proteins always form a HAP2/HAP3/HAP5 trimeric complex with DNA binding activity in mammals ${ }^{40,41}$. In Arabidopsis, CO could replace AtHAP2 in the HAP complex to form a trimetric complex, CO/AtHAP3/AtHAP5, as the CCT domain of CO and the DNA-binding domain of AtHAP2 are similar to each other ${ }^{42}$. In LD conditions, overexpression of AtHAP 2 or AtHAP 3 could delay flowering by impairing formation of the CO/AtHAP3/AtHAP5 complex, leading to decreased expression of $F T^{42}$. As a homologue of CO, Hd1 may be able to replace OsHAP2 in the HAP complex in rice. Our yeast-two-hybrid results revealed that $\mathrm{Hd} 1^{\mathrm{HXX} 74}$ could indeed interact with $\mathrm{LH} 8^{\text {Zihuiloo }}$, but not with $\mathrm{LH} 8^{\mathrm{HJX} 74}$, indicating the sequence difference, especially in the C-terminal region, between $\mathrm{LH} 8^{\text {Zihuil00 }}$ and $\mathrm{LH} 8^{\mathrm{HJX} 74}$ might be important for its interaction with $\mathrm{Hd} 1^{\mathrm{HXX} 74}$, and therefore for the regulation of rice heading (Fig. 8b). The domains responsible for the interaction between $\mathrm{LH} 8^{\mathrm{HJX} 74}$ and $\mathrm{Hd} 1^{\mathrm{HJX} 74}$ were needed to be further investigated. However, the interaction between $\mathrm{LH} 8^{\text {Zihuil00 }}$ and $\mathrm{Hd} 1^{\text {Nip }}$ (Hd1 from Nipponbare) could not be detected (data not shown), in consistent with the previous study that effects of Ghd8 depend on the genetic background ${ }^{22}$. Consequently, whether the HAP complex could be formed or not was determined by the different subunits presented in different genetic backgrounds, possibly resulting in varied functions in flowering control.

Until now, 4 genes (Ehd4, OsMADS50, OsDof12, OsPhyB) on the short arm of chromosome 3 had been identified to be involved in flowering control in rice $\mathrm{r}^{15,17-19,43,44}$. In our study, EH3 was mapped in the $750 \mathrm{~kb}$ genomic region between the Id 32 and Id 33 markers with 123 putative ORFs inside, including OsMADS50. OsMADS50, an ortholog of Arabidopsis SUPPRESSOR OF OVEREXPRSSION OF CONSTANS1 (SOC1), functions upstream of Ehd1 to indirectly 


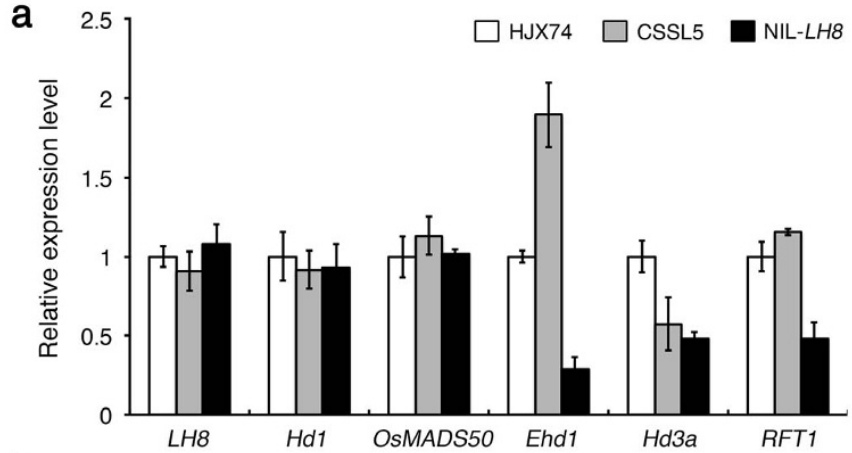

b

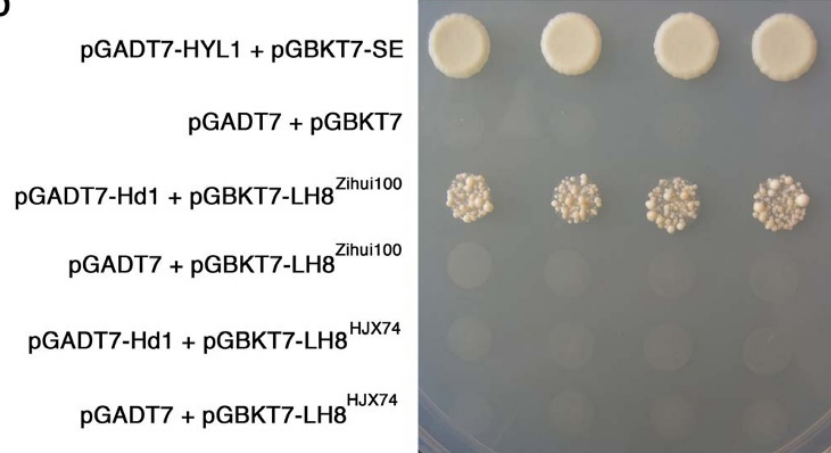

Figure 7 Characterization of the $\mathbf{L H} \mathbf{8}$ gene in rice. (a). Expression of LH8, Hd1, OsMADS50, Ehd1, Hd3a and RFT1 in HJX74 (white pillars), CSSL5 (gray pillars) and NIL-LH8 (black pillars) at dawn (for $\mathrm{LH} 8$, OsMADS50, Ehd1, Hd3a and RFT1) and dusk (for Hd1) under NSD conditions. The analysis was performed in two independent experiments. The mean values of the relative expression levels of genes in CSSL5 and NIL-LH8 compared with HJX74 are shown by cloumns, and standard deviations are indicated by the error bars. (b). Interaction of LH8 and Hd1 by yeast-two-hybrid assay. The yeast cells were grown on SD/-Leu/-Trp/His/-Ade medium. The interaction between SE and HYL1 was served as positive control, while the combination of empty prey pGADT7, and that of the empty bait vector pGBKT7 with/without LH8 from Zihuil00 were served as negative controls.

induce RFT1 transcription ${ }^{17-19}$ and promote flowering. However, no epistatic interaction had been found between LH8 and OsMADS50. Furthermore, no significant difference of the expression of OsMADS50 was found between HJX74 and CSSL5. Therefore, whether EH3 is allelic to OsMADS50 remains to be investigated. Further fine mapping and molecular identification of $E H 3$ will be necessary to provide molecular evidence for the epistatic interaction between $\mathrm{LH} 8$ and $\mathrm{EH} 3$ in regulating heading date in rice.

\section{Methods}

Plant materials and growth conditions. CSSL5 is one of a set of 1,123 chromosome segment substitution lines, which were developed from backcross progenies $\left(\mathrm{BC}_{6} \mathrm{~F}_{2}\right)$ derived from a cross between an elite indica variety from south china, HJX74, as the recurrent parent and another indica variety Zihuil00 as the donor parent.

Plants were grown under natural short-day (from July to November) and natural long-day (from March to July) conditions in a paddy field in Guangzhou $\left(23^{\circ} 07^{\prime} \mathrm{N}\right.$, $\left.113^{\circ} 15^{\prime} \mathrm{E}\right)$, China. Heading date was defined as the time when the first panicle emerged.

High-throughput genotyping using whole-genome resequencing. DNA was extracted from $100 \mathrm{mg}$ fresh rice leaves using the DNeasy Plant Mini Kit (QIAGEN Sciences). DNA was quantified using NanoDrop 1000 spectrophotometer (Thermo Scientific), and about $5 \mu \mathrm{g}$ of DNA was used for preparation of libraries for Illumina sequencing according to the protocol for the Paired-End DNA Sample Prep kit (Illumina). The libraries were used for cluster generation on a flow cell and sequenced for 76 cycles on an Illumina Genome Analyzer IIx. Base calling and filtering of lowquality bases were done using sequence control software real-time analysis, Base calling (BCL) converter and the GERALD module (Illumina).

DNA extraction and PCR amplification. Fresh leaves were collected at the seedling stage and then ground in liquid nitrogen. Microquantities of DNA were extracted from fresh leaves of each individual using a previously reported method ${ }^{45}$. Amplification was carried out on the program for the initial denaturing step with $94^{\circ} \mathrm{C}$ for $3 \mathrm{~min}$, followed by 35 cycles for $30 \mathrm{~s}$ at $94^{\circ} \mathrm{C}, 30 \mathrm{~s}$ at $55^{\circ} \mathrm{C}, 30 \mathrm{~s}$ at $72^{\circ} \mathrm{C}$, with a final extension at $72^{\circ} \mathrm{C}$ for $5 \mathrm{~min}$. PCR products were separated on $6 \%$ nondenaturing polyacrylamide gel and detected using the silver staining method ${ }^{46}$.

Linkage map and QTL analysis. We constructed the linkage map using Mapmaker/ Exp3.047. The Kosambi function was used to calculate genetic distances. QTL for heading date were estimated by Mapmaker/QTL $1.1^{48}$. Putative QTL were identified in regions exceeding 3.0 LOD (log-likelihood value).

Evaluation of genetic interactions. To analyze genetic interactions between $\mathrm{LH} 8$ and $E H 3$, we classified a total of 359 and $225 \mathrm{~F}_{2}$ plants grown under NLD and NSD conditions in 2011, respectively, into nine classes according to the genotypes of SSR markers tightly linked to the QTLs (Id83-Id82 interval for LH8, and Id32-Id33 interval for EH3). We compared the mean DTH between the nine genotypes using two-way analysis of variance (ANOVA). Testing for each genetic interaction types (AA, AD, DA and DD) was done by orthogonal contrast test in STAR software (http://bbi.irri.org/).

Mapping for $L H 8$. $\mathrm{F}_{2}$ plants with homozygous $E H 3$ allele from $\mathrm{HJX74}$ and heterozygous $L H 8$ allele from Zihuil00 were selected to generate $\mathrm{F}_{3}$ population. A total of 559 and $1,600 \mathrm{~F}_{3}$ plants were grown to fine map $L H 8$ gene under NLD and NSD conditions in 2012, respectively. Each plant was genotyped using molecular markers and phenotyped by visual examination of the first panicle emergence. The $\mathrm{F}_{4}$ recombinant lines derived from the $\mathrm{F}_{3}$ population were used for progeny testing. a

\begin{tabular}{|c|c|c|c|c|}
\hline & LH8 & $E H 3$ & Heading & $\begin{array}{l}\text { Epistatic } \\
\text { interaction }\end{array}$ \\
\hline HJX74 & $L H 8^{H J X 74}$ & $E H 3^{H J \times 74}$ & Early & No \\
\hline CSSL5 & LH8 $8^{\text {Zinui100 }}$ & $E H 3^{\text {Zihuit100 }}$ & Middle & Yes \\
\hline NIL-LH8 & LH8 zinui100 & $E H 3^{H J X 74}$ & Late & No \\
\hline NIL-EH3 & $\mathrm{LH}^{\mathrm{HJ}} \mathrm{J}$ & $E H 3^{\text {Zihuitoo }}$ & Early & No \\
\hline
\end{tabular}

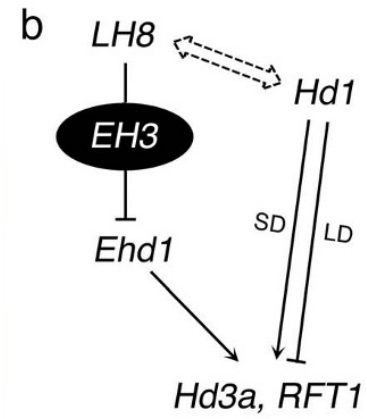

Hd3a, RFT1

Flowering

Figure 8 Schematic representation of the $\mathrm{LH} 8$ and $\mathrm{EH} 3$ mediated flowering pathway under natural growth conditions. (a). Epistatic model of rice heading controlled by $L H 8$ and $E H 3$. (b). A proposed model for the flowering pathway controlled by $L H 8$ and $E H 3$ in rice under natural growth conditions. $L H 8$ could down-regulate Ehd1, which could act on $H d 3 a$ and RFT1, thus delaying flowering in rice under natural growth conditions. EH3 might function as a modifier of $L H 8$ to shorten heading date only when specific $L H 8$ background was present. The interaction between LH8 and Hd1 depended on different alleles presented in different genetic backgrounds, possibly resulting in varied functions in flowering control. 
RNA Extraction and QRT-PCR. Total RNA from 30d-old leaves were isolated using TRIZOL reagent (Invitrogen) following the manufacturer's instruction. First-strand cDNA was reverse transcribed from DNaseI-treated RNA with oligo-dT as the primer using ReverTra Ace kit (Toyobo). Gene expression was measured by QRT-PCR using the ABI 7500 system (Life technologies). The QRT-PCR was carried out in a total volume of $20 \mu \mathrm{l}$ containing $1 X$ SYBR Green Master Mix (Life technologies). We normalized the expression levels by using UBQ5 gene as internal control. Each set of experiments was repeated three times, and the relative standard curve quantification method was used to evaluate quantitative variation. The QRT-PCR procedure was conducted at $94^{\circ} \mathrm{C}$ for $3 \mathrm{~min}$, followed by 40 cycles at $94^{\circ} \mathrm{C}$ for $30 \mathrm{~s}, 58^{\circ} \mathrm{C}$ for $30 \mathrm{~s}$, and $72^{\circ} \mathrm{C}$ for $30 \mathrm{~s}$. The QRT-PCR primers were listed in Supplemental Table S3 online.

Yeast two-hybrid assay. The cDNA fragments encoding the entire putative LH8 and Hd1 proteins were PCR-amplified, verified by sequencing and cloned into NdeIEcoRI restriction sites of the MATCHMAKER two-hybrid prey vector pGADT7 or bait vector pGBKT7 (Clontech), respectively. The bait and prey constructs were cotransformed into the yeast strain $\mathrm{AH} 109$, and the transformed cells were plated on synthetic defined medium containing all essential amino acids except leucine and tryptophan (SD/-Leu/-Trp) medium and incubated at $30^{\circ} \mathrm{C}$ for $3 \mathrm{~d}$ and then diluted and applied onto $\mathrm{SD} /$-Leu/-Trp/-His/-Ade medium and $\mathrm{SD} /$-Leu/-Trp medium (as loading control) and cultured at $30^{\circ} \mathrm{C}$ for $2-3$ days. The interaction between HYL1 and SE was used as a positive control ${ }^{49}$. The primers used in vector construction were listed in Supplemental Table S3 online.

1. Kobayashi, Y. \& Weigel, D. Move on up, it's time for change--mobile signals controlling photoperiod-dependent flowering. Genes Dev 21, 2371-84 (2007).

2. Putterill, J., Laurie, R. \& Macknight, R. It's time to flower: the genetic control of flowering time. Bioessays 26, 363-73 (2004).

3. Tsuji, H., Taoka, K. \& Shimamoto, K. Regulation of flowering in rice: two florigen genes, a complex gene network, and natural variation. Curr Opin Plant Biol 14, 45-52 (2011)

4. Yano, M. et al. Hdl, a major photoperiod sensitivity quantitative trait locus in rice, is closely related to the Arabidopsis flowering time gene CONSTANS. Plant Cell 12 2473-2484 (2000)

5. Hayama, R., Yokoi, S., Tamaki, S., Yano, M. \& Shimamoto, K. Adaptation of photoperiodic control pathways produces short-day flowering in rice. Nature 422, 719-22 (2003).

6. Doi, K. et al. Ehd1, a B-type response regulator in rice, confers short-day promotion of flowering and controls $F T$-like gene expression independently of Hd1. Genes Dev 18, 926-36 (2004).

7. Komiya, R., Yokoi, S. \& Shimamoto, K. A gene network for long-day flowering activates $R F T 1$ encoding a mobile flowering signal in rice. Development 136, 3443-50 (2009).

8. Izawa, T. Adaptation of flowering-time by natural and artificial selection in Arabidopsis and rice. J Exp Bot 58, 3091-7 (2007).

9. Izawa, T., Takahashi, Y. \& Yano, M. Comparative biology comes into bloom: genomic and genetic comparison of flowering pathways in rice and Arabidopsis. Curr Opin Plant Biol 6, 113-20 (2003).

10. Komiya, R., Ikegami, A., Tamaki, S., Yokoi, S. \& Shimamoto, K. Hd3a and RFT1 are essential for flowering in rice. Development 135, 767-74 (2008).

11. Wu, C. et al. RID1, encoding a Cys2/His2-type zinc finger transcription factor, acts as a master switch from vegetative to floral development in rice. Proc Natl Acad Sci USA 105, 12915-20 (2008).

12. Park, S. J. et al. Rice Indeterminate 1 (OsId1) is necessary for the expression of Ehd1 (Early heading date 1) regardless of photoperiod. Plant J 56, 1018-29 (2008).

13. Matsubara, K. et al. Ehd2, a rice ortholog of the maize INDETERMINATE1 gene, promotes flowering by up-regulating Ehd1. Plant Physiol 148, 1425-35 (2008).

14. Matsubara, K. et al. Ehd3, encoding a plant homeodomain finger-containing protein, is a critical promoter of rice flowering. Plant J 66, 603-12 (2011).

15. Gao, H. et al. Ehd4 encodes a novel and Oryza-genus-specific regulator of photoperiodic flowering in rice. PLoS Genet 9, e1003281 (2013).

16. Kim, S. L., Lee, S., Kim, H. J., Nam, H. G. \& An, G. OsMADS51 is a short-day flowering promoter that functions upstream of Ehd1, OsMADS14, and Hd3a. Plant Physiol 145, 1484-94 (2007).

17. Lee, S., Kim, J., Han, J. J., Han, M. J. \& An, G. Functional analyses of the flowering time gene OSMADS50, the putative SUPPRESSOR OF OVEREXPRESSION OF CO 1/AGAMOUS-LIKE 20 (SOC1/AGL20) ortholog in rice. Plant J 38, 754-64 (2004).

18. Ryu, C. H. et al. OsMADS50 and OsMADS56 function antagonistically in regulating long day (LD)-dependent flowering in rice. Plant Cell Environ 32, $1412-27$ (2009).

19. Bian, X. F. et al. Heading date gene, $d$ th 3 controlled late flowering in O. Glaberrima Steud. by down-regulating Ehd1. Plant Cell Rep 30, 2243-54 (2011).

20. Xue, W. et al. Natural variation in $G h d 7$ is an important regulator of heading date and yield potential in rice. Nat Genet 40, 761-7 (2008).

21. Wei, X. et al. DTH8 suppresses flowering in rice, influencing plant height and yield potential simultaneously. Plant Physiol 153, 1747-58 (2010).

22. Yan, W. H. et al. A major QTL, Ghd8, plays pleiotropic roles in regulating grain productivity, plant height, and heading date in rice. Mol Plant 4, 319-30 (2011).

23. Dai, X. et al. $L H D 1$, an allele of $D T H 8 / G h d 8$, controls late heading date in common wild rice (Oryza rufipogon). J Integr Plant Biol 54, 790-9 (2012).
24. Andres, F., Galbraith, D. W., Talon, M. \& Domingo, C. Analysis of PHOTOPERIOD SENSITIVITY5 sheds light on the role of phytochromes in photoperiodic flowering in rice. Plant Physiol 151, 681-90 (2009).

25. Lee, Y. S. et al. OsCOL4 is a constitutive flowering repressor upstream of Ehd1 and downstream of OsphyB. Plant J 63, 18-30 (2010).

26. Peng, L. T., Shi, Z. Y., Li, L., Shen, G. Z. \& Zhang, J. L. Ectopic expression of OsLFL1 in rice represses Ehd1 by binding on its promoter. Biochem Biophys Res Commun 360, 251-6 (2007).

27. Peng, L. T., Shi, Z. Y., Li, L., Shen, G. Z. \& Zhang, J. L. Overexpression of transcription factor OsLFL1 delays flowering time in Oryza sativa. J Plant Physiol $165,876-85$ (2008)

28. Fukuoka, S., Ebana, K., Yamamoto, T. \& Yano, M. Integration of Genomics into Rice Breeding. Rice 3, 131-137 (2010).

29. Yamamoto, T., Yonemaru, J. \& Yano, M. Towards the understanding of complex traits in rice: substantially or superficially? DNA Res 16, 141-54 (2009).

30. Fukuoka, S., Nonoue, Y. \& Yano, M. Germplasm enhancement by developing advanced plant materials from diverse rice accessions. Breeding Sci 60, 509-517 (2010).

31. Zhang, G. Q. et al. The construction of a library of single segment substitution lines in rice (Oryza sativa L.). Rice Genetics Newsletter 21, 85-87 (2004).

32. Xi, Z. Y. et al. Development of a wide population of chromosome single-segment substitution lines in the genetic background of an elite cultivar of rice (Oryza sativa L.). Genome 49, 476-84 (2006).

33. Yu, S. B. et al. Identification of quantitative trait loci and epistatic interactions for plant height and heading date in rice. Theor Appl Genet 104, 619-625 (2002).

34. Lin, H. X., Liang, Z. W., Sasaki, T. \& Yano, M. Fine mapping and characterization of quantitative trait loci $H d 4$ and $H d 5$ controlling heading date in rice. Breeding Sci 53, 51-59 (2003).

35. Gu, X. Y. \& Foley, M. E. Epistatic interactions of three loci regulate flowering time under short and long daylengths in a backcross population of rice. Theor Appl Genet 114, 745-54 (2007)

36. Uwatoko, N. et al. Epistasis among the three major flowering time genes in rice: coordinate changes of photoperiod sensitivity, basic vegetative growth and optimum photoperiod. Euphytica 163, 167-175 (2008).

37. Liu, S. et al. Genetic analysis and fine mapping of $L H 1$ and $L H 2$, a set of complementary genes controlling late heading in rice (Oryza sativa L.). Breeding Sci 62, 310-319 (2012).

38. Maity, S. N. \& de Crombrugghe, B. Role of the CCAAT-binding protein CBF/NF$\mathrm{Y}$ in transcription. Trends Biochem Sci 23, 174-8 (1998).

39. Mantovani, R. The molecular biology of the CCAAT-binding factor NF-Y. Gene 239, 15-27 (1999).

40. Goda, H. et al. Nuclear translocation of the heterotrimeric CCAAT binding factor of Aspergillus oryzae is dependent on two redundant localising signals in a single subunit. Arch Microbiol 184, 93-100 (2005).

41. Tuncher, A., Sprote, P., Gehrke, A. \& Brakhage, A. A. The CCAAT-binding complex of eukaryotes: evolution of a second NLS in the HapB subunit of the filamentous fungus Aspergillus nidulans despite functional conservation at the molecular level between yeast, A. nidulans and human. J Mol Biol 352, 517-33 (2005).

42. Wenkel, S. et al. CONSTANS and the CCAAT box binding complex share a functionally important domain and interact to regulate flowering of Arabidopsis. Plant Cell 18, 2971-84 (2006).

43. Takano, M. et al. Distinct and cooperative functions of phytochromes $A, B$, and $C$ in the control of deetiolation and flowering in rice. Plant Cell 17, 3311-25 (2005).

44. Li, D. et al. Functional characterization of rice OsDof12. Planta 229, 1159-69 (2009).

45. Shan, J. X., Zhu, M. Z., Shi, M., Gao, J. P. \& Lin, H. X. Fine mapping and candidate gene analysis of spd6, responsible for small panicle and dwarfness in wild rice (Oryza rufipogon Griff.). Theor Appl Genet 119, 827-36 (2009).

46. Li, W. T., Zeng, R. Z., Zhang, Z. M. \& Zhang, G. Q. Mapping of $S-b$ locus for F1 pollen sterility in cultivated rice using PCR based markers. Acta Botanica Sinica 44, 463-467 (2002)

47. Lander, E. S. et al. MAPMAKER: an interactive computer package for constructing primary genetic linkage maps of experimental and natural populations. Genomics 1, 174-81 (1987).

48. Lincoln, S., Daly, M. \& Lander, E. Mapping genes controlling quantitative traits with MAPMAKER/QTL1.1. 2nd edition (Whitehead Institute Technical Report, Cambridge, Massachusetts, 1992).

49. Yang, L., Liu, Z., Lu, F., Dong, A. \& Huang, H. SERRATE is a novel nuclear regulator in primary microRNA processing in Arabidopsis. Plant J 47, 841-50 (2006).

\section{Acknowledgments}

This study was finacially supported by grants from the National Natural Science Foundation of P.R. China (31200905), the Guangdong Provincial Natural Science Foundation of China (10451064201005483), the Specialized Research Fund for the Doctoral Program of Higher Education (20104404120005), and the $40^{\text {th }}$ Scientific Research Foundation for the Returned Overseas Chinese Scholars, State Education Ministry. 


\section{Author contributions}

Z.Q.L. and G.Q.Z. designed the experiments. Z.Q.L. wrote the manuscript. J.B.C. and M.Q. performed QTL analysis. J.B.C. and X.Y.L. performed map-based cloning. C.C. and H.T.Z. performed sequencing analysis. Y.H.W. performed real-time RT-PCR. R.Z.Z. and X.L.F. performed yeast-two-hybrid analysis. All authors commented on the manuscript.

\section{Additional information}

Supplementary information accompanies this paper at http://www.nature.com/ scientificreports

Competing financial interests: The authors declare no competing financial interests.
How to cite this article: Chen, J.B. et al. Characterization of Epistatic Interaction of QTLs LH8 and EH3 Controlling Heading Date in Rice. Sci. Rep. 4, 4263; DOI:10.1038/srep04263 (2014).

This work is licensed under a Creative Commons Attribution 3.0 Unported license. To view a copy of this license, visit http://creativecommons.org/licenses/by/3.0 